International Mathematical Forum, Vol. 8, 2013, no. 35, 1727 - 1734

HIKARI Ltd, www.m-hikari.com

http://dx.doi.org/10.12988/imf.2013.39169

\title{
Solitary Wave and Kink Wave Solutions for a Class of Nonlinear Evolution Equations
}

\author{
Jing $\mathbf{N a}^{1}$ \\ College of Statistics and Mathematics \\ Yunnan University of Finance and Economics \\ Kunming, Yunnan 650221, China
}

Copyright (c) 2013 Jing Na. This is an open access article distributed under the Creative Commons Attribution License, which permits unrestricted use, distribution, and reproduction in any medium, provided the original work is properly cited.

\begin{abstract}
By using the bifurcation theory of planar dynamical systems to a class of nonlinear evolution equations, the existence of traveling wave solutions is showed. Under different parametric conditions, various sufficient conditions to guarantee the existence of the above solutions are given.
\end{abstract}

Keywords: traveling wave solutions; solitary wave; kink wave and antikink wave; bifurcation of phase portraits

\section{Introduction}

In 1990, Yasunori Nejoh [4] derived the following class of nonlinear evolution equations:

$$
\wp\left(\frac{\partial \phi}{\partial \eta}\right)^{2}=c_{1} \phi^{2}+\frac{4}{5} c_{2} \phi^{\frac{5}{2}}+\frac{2}{3} c_{3} \phi^{3}+\frac{4}{7} c_{4} \phi^{\frac{7}{2}}
$$

under the boundary conditions $\phi, \phi_{\eta}, \phi_{\eta \eta} \rightarrow 0$ as $|\eta| \rightarrow 0$, where $\eta=x-\lambda t, \lambda$ denotes the velocity of moving frame, and $\wp, c_{1}, c_{2}, c_{3}, c_{4}$ are arbitrary constant parameters. (1) is a model equation which describes the one-dimensional wave

\footnotetext{
${ }^{1}$ The work is supported by the Scientific Research Foundation of Department of Education of Yunnan Province (2012Y112).
} 
propagation of ion acoustic wave in a maganetised plasma with trapped electrons

In this paper, we shall consider the bifurcation behavior of traveling wave solutions of (1) in their parametric spaces and give some solitary wave, kink and anti-kink wave solutions. To find solutions of (1), we set

$$
\phi(\eta)=\psi^{2}(\eta) .
$$

Then, (1) becomes

$$
\left(\psi_{\eta}\right)^{2}=\psi^{2}\left(a_{1}-a_{2} \psi-a_{3} \psi^{2}-a_{4} \psi^{3}\right)
$$

where the parameters $a_{1}, a_{2}, a_{3}$ and $a_{4}$ stand for

$$
a_{1}=\frac{c_{1}}{4 \wp}, \quad a_{2}=-\frac{c_{2}}{5 \wp}, \quad a_{3}=-\frac{c_{3}}{6 \wp}, \quad a_{4}=-\frac{c_{4}}{7 \wp} .
$$

We consider the following planar Hamiltonian system

$$
\psi^{\prime}(\eta)=y, \quad y^{\prime}(\eta)=a_{1} \psi-\frac{3}{2} a_{2} \psi^{2}-2 a_{3} \psi^{3}-\frac{5}{2} a_{4} \psi^{4},
$$

with the Hamiltonian

$$
H(\psi, y)=\frac{1}{2} y^{2}-\frac{1}{2} \psi^{2}\left(a_{1}-a_{2} \psi-a_{3} \psi^{2}-a_{4} \psi^{3}\right)=h .
$$

Clearly, (3) corresponds to the case of the integral constant $h=0$. Namely, if we find the dynamics of orbit defined by $H(\psi, y)=0$, then we obtain the travelling wave solution of (1).

Because we are interesting the existence of the solitary wave and kink wave solutions of (1) by the given boundary conditions. We always assume that the equilibrium $(0,0)$ is not a center, i.e., the condition $a_{1} \geq 0$ holds. For this aim, the method of bifurcation theory of dynamical systems is very useful (see [1], $[2],[3])$ in our study.

The rest of this paper is organized as follows. In Section 2, we discuss bifurcations of phase portraits of (5), where explicit parametric conditions will be derived in the case $a_{4} \neq 0$. In Section 3, we show the existence of solitary wave, kink wave and anti-kink wave solutions of (1) in the case $a_{4} \neq 0$.

\section{Bifurcation set and phase portraits of the Hamiltonian system (5)}

In this section, we shall study the bifurcation set and phase portraits of the Hamiltonian system $(5)$ in the case $a_{4} \neq 0$. 
I. Suppose that $a_{4}>0$.

In this case, making the parametric transformations

$$
\frac{a_{1}}{a_{4}}=a, \quad \frac{a_{2}}{a_{4}}=b, \quad \frac{a_{3}}{a_{4}}=c,
$$

(5) becomes

$$
\psi^{\prime}(\eta)=y, \quad y^{\prime}(\eta)=\frac{5}{2} a_{4} \psi\left(\frac{2}{5} a-\frac{3}{5} b \psi-\frac{4}{5} c \psi^{2}-\psi^{3}\right),
$$

which has the Hamiltonian

$$
H_{2}(\psi, y)=\frac{1}{2} y^{2}-\frac{1}{2} a_{4} \psi^{2}\left(a-b \psi-c \psi^{2}-\psi^{3}\right)=h .
$$

Clearly, on the $(\psi, y)$-phase plane, the abscissas $\psi_{j}$ of equilibrium points $\left(\psi_{j}, 0\right)$ of system $(7)$ are the zeros of

$$
f_{2}(\psi)=\frac{2}{5} a-\frac{3}{5} b \psi-\frac{4}{5} c \psi^{2}-\psi^{3},
$$

and zero. Denote that $\psi_{0}=0$.

Notice that

$$
f_{2}^{\prime}(\psi)=-\frac{3}{5} b-\frac{8}{5} c \psi-3 \psi^{2},
$$

which has two zeros at

$$
\psi_{1,2}^{*}=\frac{1}{15}\left(-4 c \pm \sqrt{16 c^{2}-45 b}\right)
$$

if $\Delta=16 c^{2}-45 b \geq 0$.

Let $\left(\psi_{j}, 0\right)$ be an equilibrium point of $(7)$. At this point, the determinant of the linearized system of (7) has the form

$$
J\left(\psi_{j}, 0\right)=-\frac{5}{2} a_{4}\left(\psi_{j} f_{2}^{\prime}\left(\psi_{j}\right)+f_{2}\left(\psi_{j}\right)\right) .
$$

Suppose that

$$
f_{2}(\psi)=\frac{2}{5} a-\frac{3}{5} b \psi-\frac{4}{5} c \psi^{2}-\psi^{3}=\left(\psi_{1}-\psi\right)\left(\psi_{2}-\psi\right)\left(\psi_{3}-\psi\right),
$$

where $\psi_{i}, i=1,2,3$ are real numbers.

1. $b=0$ i.e., $a_{2}=0$.

In this case, $\psi_{1}^{*}=0, \psi_{2}^{*}=-\frac{8}{15} c$. On the $(c, a)$-upper-half parametric plane, there are three bifurcation curves:

$$
L_{1}: a=0 ; \quad L_{2}: a=\frac{128}{675} c^{3}, \quad(c>0) ; \quad L_{3}: a=\frac{4}{27} c^{3}, \quad(c>0) .
$$


By the theory of planar dynamical systems, we know that if $J\left(\psi_{i}, 0\right)>0$ or $(<0)$, then the equilibrium $\left(\psi_{i}, 0\right)$ is a center (or a saddle point); if $J\left(\psi_{i}, 0\right)=0$ and the Poincare index of $\left(\psi_{i}, 0\right)$ is zero, then $\left(\psi_{i}, 0\right)$ is a cusp. By using the above facts to do qualitative analysis, under different parameter conditions, we obtain the following bifurcations of phase portraits of system (7) shown in Fig.1.

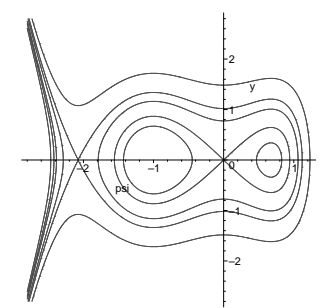

$(1-1)$

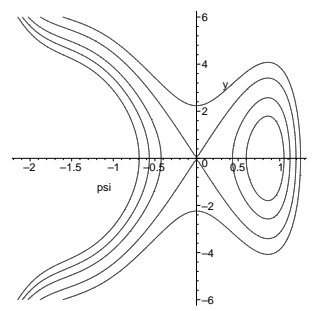

$(1-5)$

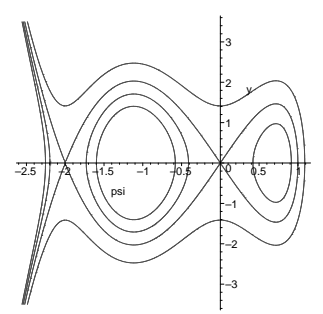

$(1-2)$

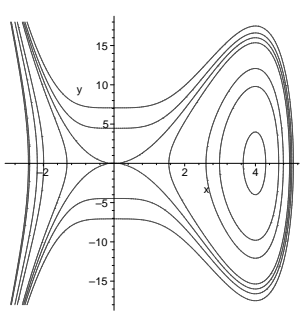

$(1-6)$

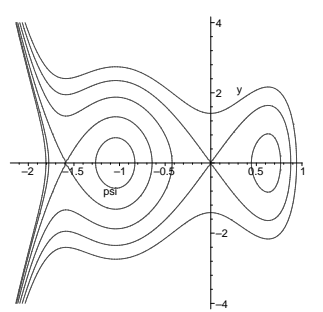

$(1-3)$

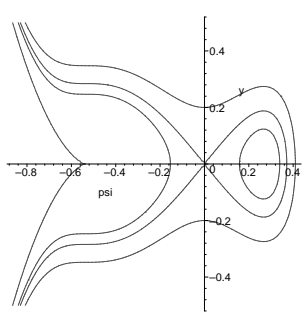

$(1-4)$

Fig. 1 The bifurcation set and bifurcations of phase portraits of (7) for $a_{2}=0$.

(1-1) $0<a<\frac{4}{27} c^{3}, c>0$. (1-2) $a=\frac{4}{27} c^{3}, c>0$. (1-3) $\frac{4}{27} c^{3}<a<\frac{128}{675} c^{3}, c>0$. (1-4) $a=\frac{128}{675} c^{3}, c>0$. (1-5) $a>\frac{128}{675} c^{3}, c>0$. or $a>0, c<0$. (1-6) $a=0, c<0$.

2. $b=\frac{a_{2}}{a_{4}}>0$ i.e., $a_{2}>0$.

On the $(c, a)$-upper-half parameter plane, there are four bifurcation curves:

$$
\begin{aligned}
& L_{1}: a=-\frac{2}{5} b c+\frac{64}{675} c^{3}-\frac{16 c^{2}-45 b}{675} \sqrt{16 c^{2}-45 b}, \quad\left(-\frac{3 \sqrt{5 b}}{4}<c<-\frac{\sqrt{15 b}}{2}\right) \\
& L_{2}: a=-\frac{2}{5} b c+\frac{64}{675} c^{3}+\frac{16 c^{2}-45 b}{675} \sqrt{16 c^{2}-45 b}, \quad\left(c>\frac{\sqrt{15 b}}{2}, c<-\frac{3 \sqrt{5 b}}{4}\right) \\
& L_{3}: a=-\frac{1}{3} b c+\frac{2}{27} c^{3}+2 \frac{c^{2}-3 b}{27} \sqrt{c^{2}-3 b}, \quad(c \geq 2 \sqrt{b}, c<-\sqrt{3 b}) \\
& L_{4}: a=0 .
\end{aligned}
$$

These bifurcation curves partition the $(c, a)$-upper half plane into five regions: $\left(A_{1}\right)-\left(A_{5}\right)$ (see Fig.2). 


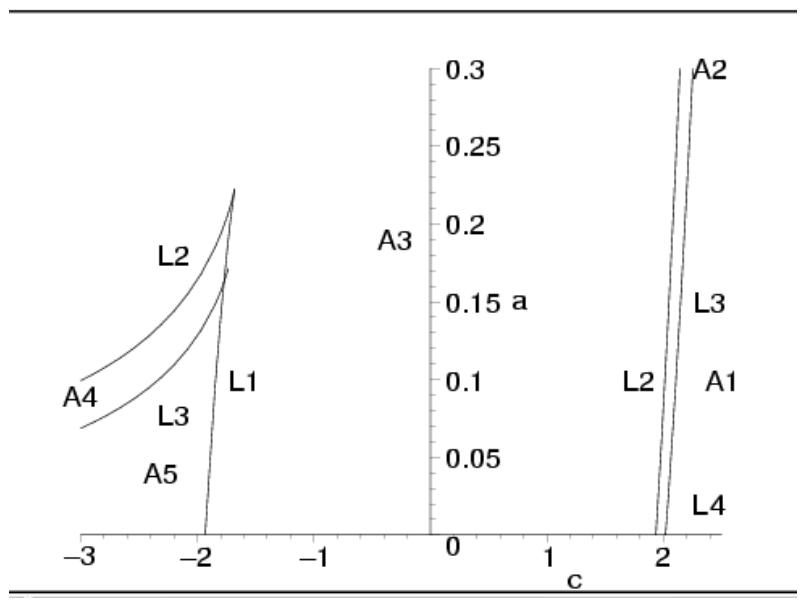

Fig. 2 The bifurcation curves of (7) for $a_{2}>0$.

Similar to the above discussion, we obtain the following results. The bifurcations of phase portraits of (7) are shown in Fig. 3.

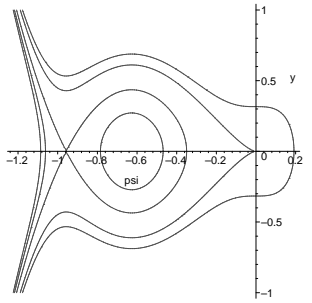

$(3-1)$

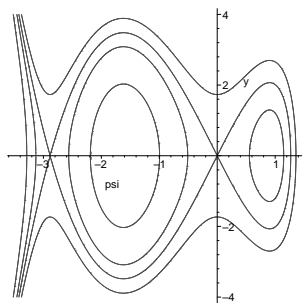

$(3-5)$

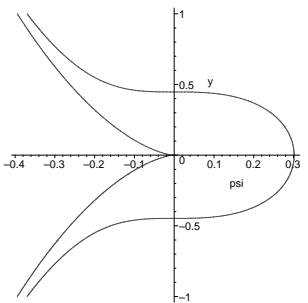

$(3-9)$

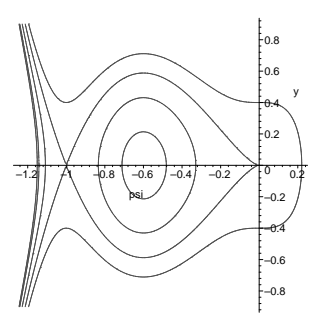

$(3-2)$

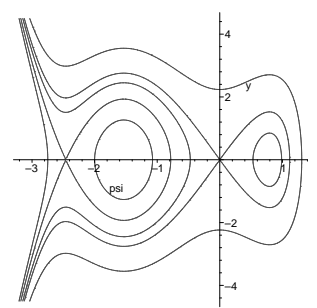

$(3-6)$

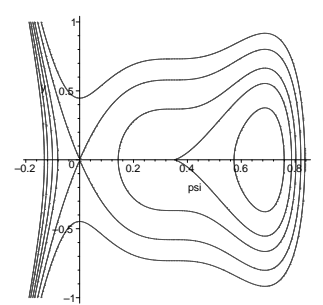

$(3-10)$

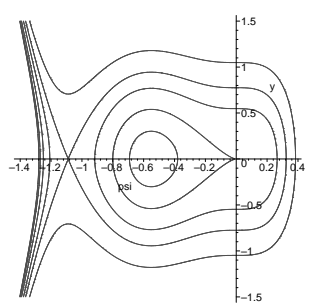

$(3-3)$

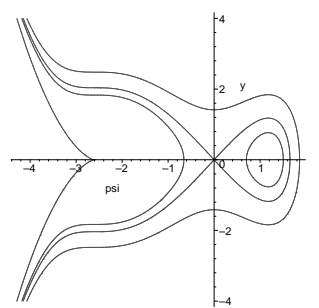

$(3-7)$

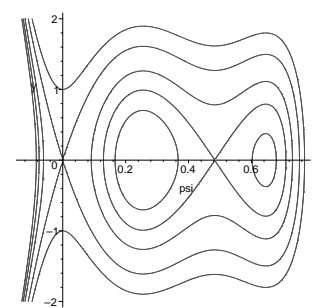

$(3-11)$

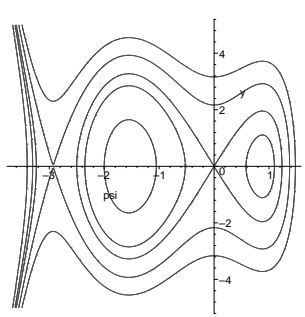

(3-4)

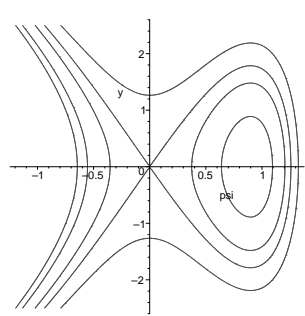

$(3-8)$

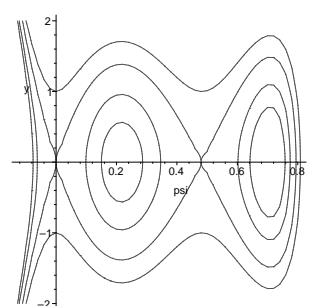

(3-12) 


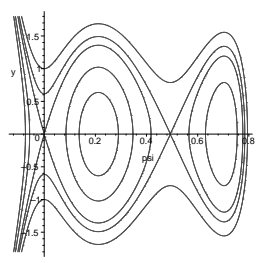

(3-13)

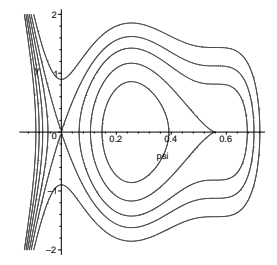

(3-14)

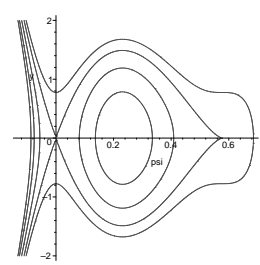

$(3-15)$

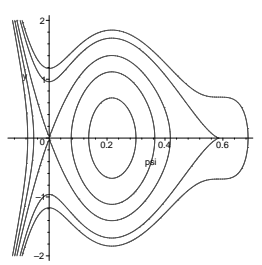

$(3-16)$

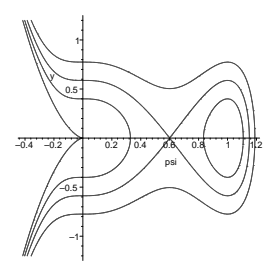

$(3-17)$

Fig. 3 The bifurcations of phase portraits of (7) for $a_{2}>0$.

(3-1) $(c, a) \in L_{4}, \frac{\sqrt{15 b}}{2}<c<2 \sqrt{b} . \quad(3-2)(c, a) \in L_{4}, c=2 \sqrt{b} . \quad(3-3)(c, a) \in$ $L_{4}, c>2 \sqrt{b}$. (3-4) $(c, a) \in A_{1}$. (3-5) $(c, a) \in L_{3}, c>2 \sqrt{b}$. (3-6) $(c, a) \in A_{2}$. (37) $(c, a) \in L_{2}, c>\frac{\sqrt{15 b}}{2}$. (3-8) $(c, a) \in A_{3}$. (3-9) $(c, a) \in L_{4},-\frac{\sqrt{15 b}}{2}<a<\frac{\sqrt{15 b}}{2}$. $(3-10)(c, a) \in L_{2}, c<-\frac{3 \sqrt{5 b}}{4}$. (3-11) $(c, a) \in A_{4}$. (3-12) $(c, a) \in L_{3}, c<-\sqrt{3 b}$. $(3-13)(c, a) \in A_{5} . \quad(3-14)(c, a) \in L_{1},-\sqrt{3 b}<c<-\frac{3 \sqrt{5 b}}{4} . \quad(3-15)(c, a) \in$ $L_{1}, c=-\sqrt{3 b}$. (3-16) $(c, a) \in L_{1},-\frac{\sqrt{15 b}}{2}<c<-\sqrt{3 b}$. (3-17) $(c, a) \in L_{4}, c<$ $-\frac{\sqrt{15 b}}{2}$.

3. $b=\frac{a_{2}}{a_{4}}<0$ i.e., $a_{2}<0$.

In this case, on the $(c, a)$-upper-half parameter plane, there are three bifurcation curves:

$$
\begin{aligned}
& L_{1}: a=-\frac{2}{5} b c+\frac{64}{675} c^{3}+\frac{16 c^{2}-45 b}{675} \sqrt{16 c^{2}-45 b} \\
& L_{2}: a=-\frac{1}{3} b c+\frac{2}{27} c^{3}+\frac{2 c^{2}-6 b}{27} \sqrt{c^{2}-3 b} ; \\
& L_{3}: a=0 .
\end{aligned}
$$

These bifurcation curves partition the $(c, a)$-upper half plane into three regions: $\left(B_{1}\right)-\left(B_{3}\right)$ (see Fig. 4$)$.

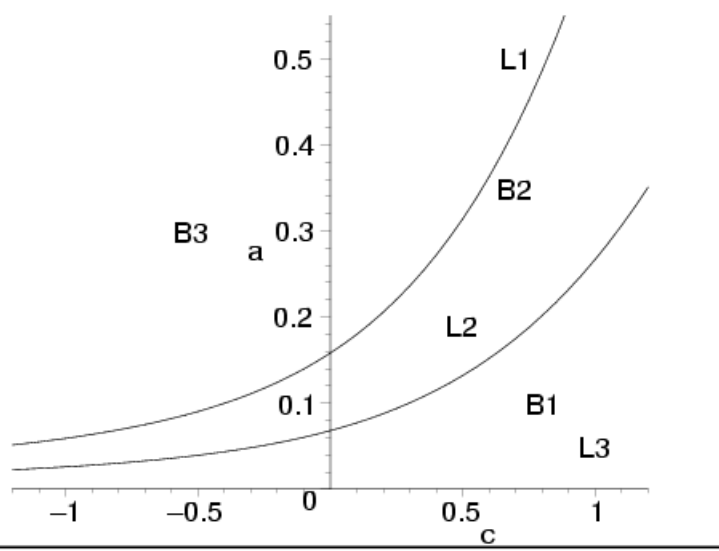

Fig. 4 The bifurcation curves of (7)for $a_{2}<0$. 
Thus, we obtain the bifurcations of phase portraits of (7) as follows.

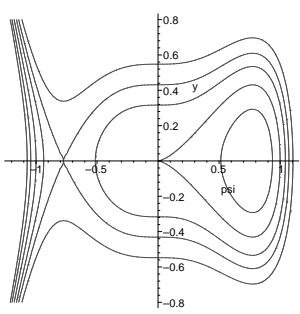

$(5-1)$

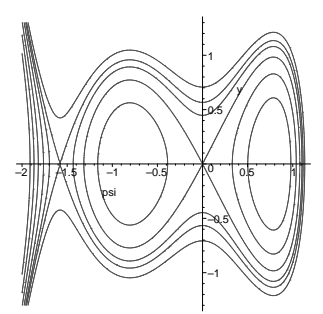

$(5-2)$

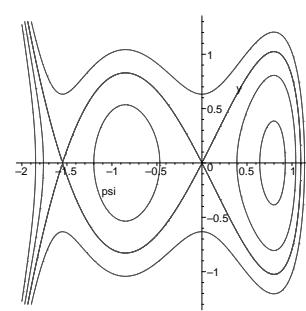

$(5-3)$

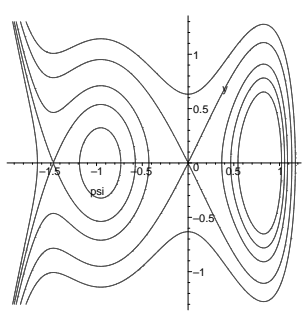

$(5-4)$

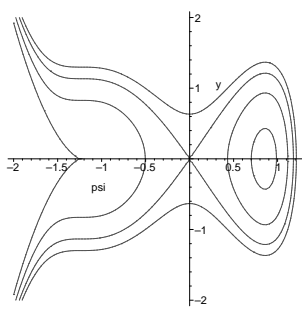

$(5-5)$

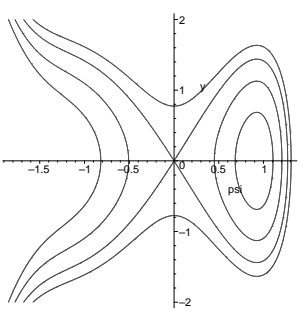

$(5-6)$

Fig. 5 The bifurcations of phase portraits of (7) for $a_{2}<0$.

$(5-1)(c, a) \in L_{3} .(5-2)(c, a) \in B_{1} . \quad(5-3)(c, a) \in L_{2} .(5-4)(c, a) \in B_{2} .(5-5)$ $(c, a) \in L_{1}$. (5-6) $(c, a) \in B_{3}$.

II. Suppose that $a_{4}<0$. Letting

$$
\psi \rightarrow-\omega, \quad \eta \rightarrow-\gamma, \quad y \rightarrow y,
$$

then we have

$$
\frac{\mathrm{d} \omega}{\mathrm{d} \gamma}=y, \quad \frac{\mathrm{d} y}{\mathrm{~d} \gamma}=a_{1} \omega+\frac{3}{2} a_{2} \omega^{2}-2 a_{3} \omega^{3}-\frac{5}{2}\left(-a_{4}\right) \omega^{4} .
$$

This implies that system (9) has the same bifurcation set and phase portraits as system $(7)$ in the case $a_{4}>0$.

\section{$3 \quad$ Solitary wave and kink wave solutions of (1).}

For the case $a_{4} \neq 0$, it is not possible to obtain exact explicit parametric representation of solitary wave and kink wave solutions of (1). From the discussion of section 2 , we have the following results.

Theorem 3.1

1. Equation (1) has always at least one solitary wave solution of peak form, under the following parameter conditions:

(1) $a_{2}=0$ (see Fig.1(1-1)-(1-6)); 
(2) $a_{2}>0$ (see Fig.3 (3-3)-(3-8), (3-10)-(3-14) and (3-16)-(3-17));

(3) $a_{2}<0$ (see Fig.5(5-1)-(5-6)).

2. Equation (1) has a solitary wave solution of peak form and a solitary wave solution of valley form, under the following parameter conditions:

(1) $0<a<\frac{4}{27} c^{3}, c>0$ (see Fig. 1(1-1));

(2) $(c, a) \in A_{1}$ (see Fig. 3(3-4));

(3) $(c, a) \in B_{1}$ (see Fig. 5(5-2)).

3. Equation (1) has the coexistence of a solitary wave solution of peak form, a kink wave and an anti-kink wave solution, under the following parameter conditions:

(1) $a=\frac{4}{27} c^{3}, c>0$ (see Fig. 1(1-2));

(2) $(c, a) \in L_{3}, c>2 \sqrt{b}$ (see Fig. $\left.3(3-5)\right)$;

(3) $(c, a) \in L_{2}, c<-\frac{3 \sqrt{5 b}}{4}$ (see Fig. $\left.3(3-12)\right)$;

(4) $(c, a) \in L_{2}$ (see Fig. 5(5-3)).

4. Equation (1) has a kink wave solution and an anti-kink wave solution, under the following parameter conditions:

(1) $(c, a) \in L_{4}, c=2 \sqrt{b}$ (see Fig. $3(3-2)$ );

(2) $(c, a) \in L_{1},-3 \sqrt{b}<c<-\frac{3 \sqrt{5 b}}{4}$ (see Fig. 3(3-15)).

\section{References}

[1] S.N. Chow, J.K. Hale, Method of Bifurcation Theory, Springer-Verlag, New York, 1982.

[2] Jibin Li, Zhengrong Liu , Traveling wave solutions for a class of nonlinear dispersive equations, Chinese Ann. Math. Ser B, 23(3)(2002), 397-418.

[3] Yan Yan, Wenquan Wang, Bifurcations of Traveling Wave Solutions for a Class of Camassa - Holm Equations, Journal of Yunnan Nationalities University, 15(3)(2006), 178-179.

[4] Nejoh Yasunori, New spliky waves explosive modes and periodic progoressive waves in a magnetised plasma, J. Phys. A: Math. Gen., 23(1990), 1973-1984.

\section{Received: September 1, 2013}

